\title{
ResearchGate
}

See discussions, stats, and author profiles for this publication at: https://www.researchgate.net/publication/262920007

\section{On feckly clean rings}

ARTICLE · JUNE 2014

Source: arXiv

CITATION

1

3 AUTHORS, INCLUDING:

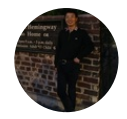

Huanyin Chen

Hangzhou Normal University

291 PUBLICATIONS 771 CITATIONS

SEE PROFILE
READS

58 


\title{
ON FECKLY CLEAN RINGS
}

\author{
H. CHEN, H. KOSE, AND Y. KURTULMAZ
}

\begin{abstract}
A ring $R$ is feckly clean provided that for any $a \in R$ there exists an element $e \in R$ and a full element $u \in R$ such that $a=e+u, e R(1-e) \subseteq J(R)$. We prove that a ring $R$ is feckly clean if and only if for any $a \in R$, there exists an element $e \in R$ such that $V(a) \subseteq V(e), V(1-a) \subseteq V(1-e)$ and $e R(1-e) \subseteq J(R)$, if and only if for any distinct maximal ideals $M$ and $N$, there exists an element $e \in R$ such that $e \in M, 1-e \in N$ and $e R(1-e) \subseteq J(R)$, if and only if $J-\operatorname{spec}(R)$ is strongly zero dimensional, if and only if $\operatorname{Max}(R)$ is strongly zero dimensional and every prime ideal containing $J(R)$ is contained in a unique maximal ideal. More explicit characterizations are also discussed for commutative feckly clean rings.
\end{abstract}

2010 MSC: Primary 16S50, 16U99; Secondary 16S34, 16 U60.

Key words: feckly clean ring; strongly zero dimensional space; pm-ring.

\section{INTRODUCTION}

An element $a$ of a ring $R$ is clean provided that $a$ is the sum of an idempotent and a unit in $R$. A ring $R$ is clean provided that every element in $R$ is clean. Clean rings are defined by Nicholson, and classes of rings that share properties with clean rings are of great interest to many researchers (cf. [1-3], [6], [8-10] and [14]). The motivation of this article is to consider such a kind of rings and characterize them in terms of topological properties. An element $u \in R$ is said to be a full element if $R u R=R$. We say that an element $a \in R$ is feckly clean provided that there exists an element $e \in R$ and a full element $u \in R$ such that $a=e+u, e R(1-e) \subseteq J(R)$. A ring $R$ is feckly clean provided that every element in $R$ is feckly clean. It is easily shown that a ring $R$ is feckly clean if and only if $R / J(R)$ is feckly clean. Clearly, every abelian clean element is feckly clean. Thus, abelian clean rings are feckly clean. But the converse is not true.

\footnotetext{
${ }^{1}$ Department of Mathematics, Hangzhou Normal University, Hangzhou 310034, China, huanyinchen@aliyun.com.

${ }^{2}$ Department of Mathematics, Ahi Evran University, Kirsehir, Turkey, handankose@gmail.com.

${ }^{3}$ Department of Mathematics, Bilkent University, Ankara, Turkey, yosum@fen.bilkent.edu.tr .
} 
Example 1.1. Let $R=\left\{\frac{m}{n} \mid m, n \in \mathbb{Z},(m, n)=1\right.$ and $\left.2,3 \nmid n\right\}$. Then $\operatorname{Max}(R)=\{2 R, 3 R\}$, and so $R / J(R) \cong \mathbb{Z}_{2} \oplus \mathbb{Z}_{3}$. Hence, $R / J(R)$ is clean, and so $R$ is a commutative feckly clean ring. As idempotents do not lift modulo $J(R)$, we see that $R$ is not clean. In fact, $4 \in R$ can not be written as the sum of an idempotent and a unit.

Also right quasi-duo exchange rings are feckly clean. Some other examples will be also provided. We will show that feckly clean rings are mostly characterized by using the topological spaces of all maximal ideals of $R$, and of all prime ideals containing the Jacobson radical of $R$.

In Section 2, we show that a ring $R$ is feckly clean if and only if for any $a \in R$, there exists an element $e \in R$ such that $V(a) \subseteq V(e), V(1-a) \subseteq V(1-e)$ and $e R(1-e) \subseteq J(R)$, if and only if for any distinct maximal ideals $M$ and $N$, there exists an element $e \in R$ such that $e \in M, 1-e \in N$ and $e R(1-e) \subseteq J(R)$. In Section 3 , we consider feckly clean in terms of the topological space of all prime ideals containing the Jacobson radical of $R$. It is shown that a ring $R$ is feckly clean if and only if $J-\operatorname{spec}(R)$ is strongly zero dimensional, if and only if $\operatorname{Max}(R)$ is strongly zero dimensional and every prime ideal containing $J(R)$ is contained in a unique maximal ideal. In Section 4, we prove that every right (left) quasi-duo ring is feckly clean, and provide more examples of such rings. In the last section, more explicit characterizations are also discussed for commutative feckly clean rings.

Throughout, all rings are associative with an identity. $\operatorname{Max}(R)$ denote the denote the topological space of all maximal ideals of $R$. We always use $J$-spec $(R)$ to stand for the topological space of all prime ideals of $R$ that containing the Jacobson radical. $J(R)$ will denote the Jacobson radical of $R$ and $U(R)$ will be the set of invertible element in $R$.

\section{Separative Properties}

Let $R$ be a ring. Define the maximal spectrum of $R$ as the set of all maximal ideals of $R$ which we denote as $\operatorname{Max}(R)$. Let $I$ be an ideal of $R$, and let $E(I)=$ $\{P \in \operatorname{Max}(R) \mid I \nsubseteq P\}$. Then $E(R)=\operatorname{Max}(R), E(0)=\emptyset, E(I) \cap E(J)=E(I J)$ and $\bigcup_{i} E\left(I_{i}\right)=E\left(\sum_{i} I_{i}\right)$. So $\operatorname{Max}(R)$ is a topological space, where $\{E(I) \mid I \unlhd R\}$ is the collection of its open sets. Let $V(I)=\operatorname{Max}(R)-E(I)$. Then $V(I)=\{P \in$ $\operatorname{Max}(R) \mid I \subseteq P\}$. In addition, $V(0)=\operatorname{Max}(R), V(R)=\emptyset, V(I) \cup V(J)=V(I J)$ and $\bigcap_{i} V\left(I_{i}\right)=V\left(\sum_{i} I_{i}\right)$. Let $a \in R$, and let $V(a):=V(R a R)$. It is easy to verify that $V(I)=\bigcap_{a \in I} V(a)$. Also we use $E(a)$ to stand for $E(R a R)$. Let $S$ and 
$T$ be two sets. We always use $S \sqcup T$ to denote the set $S \cup T$ with $S \cap T=\emptyset$. In view of [4, Lemma 17.1.3], $\operatorname{Max}(R)$ is a compact space. The aim of this section is to characterize the feckly clean rings by means of the separation property of the topological space $\operatorname{Max}(R)$.

Lemma 2.1. Let $R$ be a ring, and let $P$ be a maximal ideal of $R$. Then $J(R) \subseteq P$.

Proof. Let $x \in J(R)$. If $x \notin P$, then $R x R+P=R$. Write $s_{1} x t_{1}+\cdots+s_{n} x t_{n}+y=1$ where $y \in P, s_{i}, t_{i} \in R$ for $1 \leq i \leq n$. Then $y=1-\left(s_{1} x t_{1}+\cdots+s_{n} x t_{n}\right) \in U(R)$, a contradiction. Therefore $x \in P$. Accordingly, $J(R) \subseteq P$.

Lemma 2.2. Let $R$ be a ring. Then the following are equivalent:

(1) $a \in R$ is feckly clean.

(2) There exists an element $e \in R$ such that $V(a-1) \subseteq V(e) \subseteq \operatorname{Max}(R)-$ $V(a), e R(1-e) \subseteq J(R)$.

Proof. (1) $\Rightarrow(2)$ Let $a \in R$ be feckly clean. Then there exist an $e \in R$ and a full element $u \in U(R)$ such that $a=e+u$ and $e R(1-e) \subseteq J(R)$. For any $P \in V(a-1)$, we see that $P \notin V(1-e)$; otherwise, $u=(a-1)+(1-e) \in P$. Clearly, $V(e) \cap V(1-e)=\emptyset$. For any $P \in \operatorname{Max}(R)$, as $e R(1-e) \subseteq J(R)$, we see that $e R(1-e) \subseteq P$ by Lemma 2.1. This implies that $e \in P$ or $1-e \in P$. Thus, $P \in V(e)$ or $P \in V(1-e)$. Therefore $\operatorname{Max}(R)=V(e) \bigsqcup V(1-e)$; hence, $P \in V(e)$. We infer that $V(a-1) \subseteq V(e)$. If $P \in \operatorname{Max}(R)$ and $P \notin \operatorname{Max}(R)-V(a)$, then $P \in V(a)$; hence, $P \notin V(e)$; otherwise, $u=a-e \in P$. This implies that $V(e) \subseteq \operatorname{Max}(R)-V(a)$. Therefore $V(a-1) \subseteq V(e) \subseteq \operatorname{Max}(R)-V(a)$.

$(2) \Rightarrow(1)$ Assume that there exists an element $e \in R$ such that $V(a-1) \subseteq$ $V(e) \subseteq \operatorname{Max}(R)-V(a), e R(1-e) \subseteq J(R)$ Let $u=a-e$. If $R u R \neq R$, by using Zorn's Lemma, there exists a maximal ideal $P$ such that $R u R \subseteq P \varsubsetneqq R$. In light

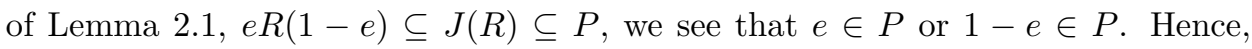
$P \in V(e)$ or $P \in V(1-e)$. If $P \in V(e)$, then $a=e+u \in P$, whence, $P \in V(a)$, a contradiction. If $P \in V(1-e)$, then $a-1=(e-1)+u \in P$, whence, $P \in V(a-1)$. Thus $P \in V(e)$, a contradiction. Therefore $a-e \in R$ is a full element, and so $a$ is feckly clean.

Theorem 2.3. Let $R$ be a ring. Then the following are equivalent:

(1) $R$ is feckly clean.

(2) For any disjoint closed sets $A$ and $B$ of $\operatorname{Max}(R)$, there exists an element $e \in R$ such that $A \subseteq V(e), B \subseteq V(1-e)$ and $e R(1-e) \subseteq J(R)$.

(3) For any $a \in R$, there exists an element $e \in R$ such that $V(a) \subseteq V(e), V(1-$ $a) \subseteq V(1-e)$ and $e R(1-e) \subseteq J(R)$. 
Proof. (1) $\Rightarrow(2)$ Let $A$ and $B$ be disjoint closed sets of $\operatorname{Max}(R)$. Then $A \bigcap B=\emptyset$. Clearly, there exist two ideals $I$ and $J$ such that $A=V(I)$ and $B=V(J)$; hence, $V(I) \cap V(J)=\emptyset$. If $I+J \neq R$, then there exists a maximal ideal $P$ of $R$ such that $I+J \subseteq P \varsubsetneqq R$. Hence, $P \in V(I+J)=V(I) \cap V(J)$, a contradiction. This implies that $I+J=R$, and so $a+b=1$ for some $a \in I$ and $b \in J$. As $a \in R$ is feckly clean, it follows from Lemma 2.2 that there exists an element $e \in R$ such that

$$
V(a-1) \subseteq V(1-e) \subseteq \operatorname{Max}(R)-V(a),(1-e) \operatorname{Re} \in J(R)
$$

It is easy to check that

$$
\begin{aligned}
& B=V(J) \subseteq V(b) \\
& =V(a-1) \subseteq V(1-e) \subseteq \operatorname{Max}(R)-V(a) \\
& \subseteq \operatorname{Max}(R)-V(I)=\operatorname{Max}(R)-A .
\end{aligned}
$$

Thus $B \subseteq V(1-e)$. As $\operatorname{Max}(R)=V(e) \sqcup V(1-e)$ and $V(1-e) \subseteq \operatorname{Max}(R)-A$, we see that $A \subseteq V(e)$, as required.

$(2) \Rightarrow(3)$ For any $a \in R, V(a) \bigcap V(1-a)=\emptyset$. That is, $V(a)$ and $V(a-1)$ are disjoint closed sets of $\operatorname{Max}(R)$. By hypothesis, there exists an element $e \in R$ such that $V(a) \subseteq V(e), V(1-a) \subseteq V(1-e)$ and $e R(1-e) \subseteq J(R)$.

$(3) \Rightarrow(1)$ For any $a \in R$, we can find an element $e \in R$ such that $V(a) \subseteq V(1-e)$ and $V(a-1) \subseteq V(e)$ with $(1-e) R e \subseteq J(R)$. If $P \in \operatorname{Max}(R)$ and $P \notin \operatorname{Max}(R)-$ $V(a)$, then $P \in V(a)$, and so $P \in V(1-e)$. This implies that $P \notin V(e)$. Hence, $V(e) \subseteq \operatorname{Max}(R)-V(a)$. Clearly, $(\operatorname{Re} R(1-e) R)^{2} \subseteq R(1-e) R e R \subseteq J(R)$. As $J(R)$ is semiprime, we see that $e R(1-e) \subseteq J(R)$. In view of Lemma 2.2, $a \in R$ is feckly clean.

Corollary 2.4. Let $R$ be a ring. Then the following are equivalent:

(1) $R$ is feckly clean.

(2) For any disjoint compact sets $A$ and $B$ of $\operatorname{Max}(R)$, there exists an element $e \in R$ such that $A \subseteq V(e), B \subseteq V(1-e)$ and $e R(1-e) \subseteq J(R)$.

Proof. (1) $\Rightarrow(2)$ Let $P, Q \in \operatorname{Max}(R), P \neq Q$. Then there exist $a \in P, b \in Q$ such that $a+b=1$. As $R$ is feckly clean, we can find an element $e \in R$ and a full element $u \in R$ such that $a=e+u$ and $e R(1-e) \subseteq J(R)$. This implies that $e \notin P$. Clearly, $b=1-a=(1-e)-u$, and so $1-e \notin Q$. Consequently, $1-e \in P$ and $e \in Q$. Thus, $P \in V(1-e)$ and $Q \in V(e)$. One easily checks that $\operatorname{Max}(R)=V(e) \bigsqcup V(1-e)$. Hence, $V(e)$ and $V(1-e)$ are clopen sets. That is, $\operatorname{Max}(R)$ is an Hausdorff space. In light of [4, Lemma 17.1.3], $\operatorname{Max}(R)$ is compact. So every compact subset of $\operatorname{Max}(R)$ is closed. For any disjoint compact subsets $A$ and $B$ of $\operatorname{Max}(R)$, it follows from Lemma 2.2 that there exists an element $e \in R$ such that $A \subseteq V(e), B \subseteq V(1-e)$ and $e R(1-e) \subseteq J(R)$. 
$(2) \Rightarrow(1)$ As $\operatorname{Max}(R)$ is compact, every closed set is compact. Thus we complete the proof by Theorem 2.3 .

Lemma 2.5. If $e, f \in R$ such that e $R(1-e), f R(1-f) \subseteq J(R)$, then there exists $g \in R$ such that $E(e) \bigcup E(f)=E(g)$ and $g R(1-g) \subseteq J(R)$.

Proof. Set $g=e+f-e f$. If $P \notin E(e) \bigcup E(f)$, then $e, f \in P$, and so $e+f-e f \in P$. This implies that $P \notin E(e+f-e f)$; hence, $E(g) \subseteq E(e) \cup E(f)$. Suppose that $P \in E(e) \cup E(f)$, then $e \notin P$ or $f \notin P$. As $e R(1-e), f R(1-f) \subseteq J(R) \subseteq P$, we see that $1-e \in P$ or $1-f \in P$. This implies that $(1-e)(1-f) \in P$, and then $1-(1-e)(1-f) \notin P$. That is, $e+f-e f \notin P$. Therefore $P \in E(g)$, and so $E(e) \bigcup E(f) \subseteq E(g)$. As a result, $E(e) \bigcup E(f)=E(g)$. On the other hand, $g R(1-g) \subseteq e R(1-e)(1-f)+(1-e) f R(1-e)(1-f) \subseteq J(R)$, as asserted.

Theorem 2.6. Let $R$ be a ring. Then the following are equivalent:

(1) $R$ is feckly clean.

(2) For any distinct maximal ideals $M$ and $N$, there exists an element $e \in R$ such that $e \in M, 1-e \in N$ and $e R(1-e) \subseteq J(R)$.

Proof. (1) $\Rightarrow(2)$ Suppose that $M$ and $N$ are distinct maximal ideals. Then $M+N=R$. Write $1=a+b$ with $a \in M, b \in N$. As $R$ is feckly clean, there exist an element $e \in R$ and a full element $u \in R$ such that $b=e+u$ and $e R(1-e) \subseteq J(R)$. Obviously, $\operatorname{Max}(R)=V(e) \bigsqcup V(1-e)$. If $M \in V(1-e)$, then $1-e \in M$. As $1-b=a \in M$, we see that $u \in M$, a contradiction. Therefore $M \in V(e)$, i.e., $e \in M$. Similarly, we show that $1-e \in N$, as desired.

$(2) \Rightarrow(1)$ Let $A$ and $B$ be disjoint closed sets of $\operatorname{Max}(R)$. Take $M \in B$. For any $N \in A, M \neq N$. By hypothesis, there exists an element $g \in R$ such that $g \in M, 1-g \in N$ and $g R(1-g) \subseteq J(R)$. Hence, $g \notin N$, and so $N \in E(g)$. As $\operatorname{Max}(R)$ is compact, and then so is $A$. Thus, we can find some $g_{1}, \cdots, g_{n} \in R$ such that $A \subseteq \bigcup_{i=1}^{n} E\left(g_{i}\right)$ and $g_{i} R\left(1-g_{i}\right) \subseteq J(R)$ for each $i$. According to Lemma 2.5, we can find an element $f \in R$ such that $\bigcup_{i=1}^{n} E\left(g_{i}\right)=E(f)$ and $f R(1-f) \subseteq J(R)$. Set $e=1-f$. Then $(1-e) R e \subseteq J(R)$. This implies that $e R(1-e) \subseteq J(R)$. Further, we see that $A \subseteq V(e)$.

Clearly, $M \in V\left(g_{1}\right)$ and $M \in V\left(g_{2}\right)$. Hence, $M \in V\left(g_{1}+g_{2}-g_{1} g_{2}\right)$. By iteration of this process, $M \in V(f)=V(1-e)$. As $B$ is compact, we can find some $e_{1}, \cdots, e_{m} \in R$ such that $A \subseteq V\left(e_{i}\right), B \subseteq \bigcup_{i=1}^{m} E\left(e_{i}\right)$ and $e_{i} R\left(1-e_{i}\right) \subseteq J(R)$ for each $i$. In light of Lemma 2.5, we can find an element $\alpha \in R$ such that $B \subseteq E(\alpha)$. 
Clearly, $V\left(e_{1}\right) \bigcap V\left(e_{2}\right) \subseteq V\left(e_{1}+e_{2}-e_{1} e_{2}\right)$. Furthermore, $A \subseteq \bigcap_{i=1}^{m} V\left(e_{i}\right)=V(\alpha)$. In addition, $\alpha R(1-\alpha) \subseteq J(R)$. According to Theorem 2.3, $R$ is feckly clean.

Corollary 2.7. If $a+b=1$ in $R$ implies that there exists an element $e \in R$ such that $1+$ ar $\in e R, 1+b s \in(1-e) R$ where $e R(1-e) \subseteq J(R)$, then $R$ is feckly clean.

Proof. Assume that $M$ and $N$ are distinct maximal ideals of $R$. Then $M+N=R$. Write $a+b=1$ with $a \in M, b \in N$. By hypothesis, there exist $r, s \in R$ and an element $e \in R$ such that $1+a r \in e R, 1+b s \in(1-e) R$, where $e R(1-e) \subseteq J(R)$. As $a \in M$, we see that $e \notin M$. As $e R(1-e) \subseteq J(R) \subseteq M$, we have $1-e \in M$. Similarly, $1-e \notin N$, and so $e \in N$. In light of Theorem 2.6, $R$ is feckly clean.

A ring $R$ is local if it has only one maximal right ideal. As is well known, a ring $R$ is local if and only if $a+b=1$ in $R$ implies that either $a$ or $b$ is invertible. We claim that every local ring $R$ is feckly clean. Given $a+b=1$ in $R$. Then either $a \in U(R)$ or $1-a \in U(R)$. If $a \in U(R)$, choosing $e=0$, then $1+a\left(-a^{-1}\right) \in e R, 1+b \cdot 0 \in(1-e) R$. If $b \in U(R)$, choosing $e=1$, then $1+a \cdot 0 \in e R, 1+b \cdot\left(-b^{-1}\right) \in(1-e) R$. Clearly, $e R(1-e) \subseteq J(R)$ in each case, and we are done.

\section{Strongly Zero-Dimensional Spaces}

A topological space $X$ is said to be strongly zero-dimensional provided that any two disjoint closed sets are separated by clopen sets, that is, if $A$ and $B$ are disjoint closed sets, then there exist disjoint clopen sets $C_{1}, C_{2}$ such that $A \subseteq C_{1}$ and $B \subseteq C_{2}$. The aim of this section is to investigate feckly clean rings by means of some strongly zero-dimensional spaces.

Let $J$-spec $(R)=\{P \in \operatorname{Spec}(R) \mid J(R) \subseteq P\}$. As is well known, the Jacobson radical $J(R)$ is semiprime, and so $J(R)$ is the intersection of some prime ideals. Hence, $J(R)=\bigcap_{P \in J-\operatorname{Spec}(R)} P$. Let $I$ be an ideal of $R$, and let $F(I)=\{P \in$ $J-\operatorname{spec}(R) \mid I \nsubseteq P\}$. Then $F(R)=J-\operatorname{spec}(R), F(0)=\emptyset, F(I) \cap F(J)=F(I J)$ and $\bigcup_{i} F\left(I_{i}\right)=F\left(\sum_{i} I_{i}\right)$. So $J$-spec $(R)$ is a topological space, where $\{F(I) \mid I \unlhd R\}$ is the collection of its open sets. Let $W(I)=J-\operatorname{spec}(R)-F(I)$. Then $W(I)=$ $\{P \in J-\operatorname{spec}(R) \mid I \subseteq P\}$ is the collection of its closed sets. We use $W(a)$ to denote $W(R a R)$ for any $a \in R$.

Lemma 3.1. Let $R$ be a ring. Then $a \in R$ is feckly clean if and only if there exists an element $e \in R$ such that $W(a-1) \subseteq W(e) \subseteq J-\operatorname{spec}(R)-W(a), e R(1-e) \subseteq$ $J(R)$. 
Proof. Let $a \in R$ be feckly clean. Then there exist an element $e \in R$ and a full element $u \in R$ such that $a=e+u$ and $e R(1-e) \subseteq J(R)$. For any $P \in J-\operatorname{spec}(R)$, as $e R(1-e) \subseteq J(R) \subseteq P$, we see that $e \in P$ or $1-e \in P$. Thus, $P \in W(e)$ or $P \in W(1-e)$. Therefore $J-\operatorname{spec}(R)=W(e) \bigsqcup W(1-e)$. As in the proof of Lemma $2.2, W(a-1) \subseteq W(e) \subseteq J-\operatorname{spec}(R)-W(a)$, as required.

The proof of the converse is the same as in Lemma 2.2 .

Lemma 3.2. Let $R$ be a ring. If $A$ is a clopen subset of $J$-spec $(R)$, then there exists an element $e \in R$ such that $A=W(e), e R(1-e) \subseteq J(R)$.

Proof. Let $A$ be a clopen subset of $J$-spec $(R)$. Then we can find ideals $I$ and $J$ of $R$ such that $A=W(I)$ and $J-\operatorname{spec}(R)=W(I) \sqcup W(J)$. Thus, $W(I J)=$ $J$-spec $(R)$. Hence $I J \subseteq \bigcap_{P \in \operatorname{Spec}(R)}\{P \mid J(R) \subseteq P\}=J(R)$. On the other hand, $W(I) \bigcap W(J)=\emptyset$, and so $W(I+J)=\emptyset$. If $I+J \neq R$, then there exists a maximal ideal $P$ of $R$ such that $I+J \subseteq P \varsubsetneqq R$. In light of Lemma 2.1, $J(R) \subseteq P$, and so $P \in J$-spec $(R)$; hence, $P \in W(I+J)$, a contradiction. Thus, $I+J=R$. Write $1=e+f, e \in I, f \in J$. Then

$$
J-\operatorname{spec}(R)=W(e) \sqcup W(f), W(I) \subseteq W(e), W(J) \subseteq W(f) .
$$

It follows from $J$-spec $(R)=W(I) \sqcup W(J)$ that $W(I)=W(e)$, as desired.

Theorem 3.3. A ring $R$ is feckly clean if and only if $J$-spec $(R)$ is strongly zero dimensional.

Proof. Suppose that $R$ is feckly clean. Let $A$ and $B$ be disjoint closed sets of $J$-spec $(R)$. Then $A \cap B=\emptyset$. Clearly, there exist two ideals $I$ and $J$ such that $A=W(I)$ and $B=W(J)$. It is easy to verify that $I+J=R$. Write $a+b=1$ with $a \in I$ and $b \in J$. Since $a \in R$ is feckly clean, by virtue of Lemma 3.1, there exists an element $e \in R$ such that

$$
W(a-1) \subseteq W(1-e) \subseteq J-\operatorname{spec}(R)-W(a),(1-e) R e \subseteq J(R) .
$$

Clearly,

$$
\begin{aligned}
& B=W(J) \subseteq W(b) \\
& =W(a-1) \subseteq W(1-e) \subseteq J-\operatorname{spec}(R)-W(a) \\
& \subseteq J-\operatorname{spec}(R)-W(I)=J-\operatorname{spec}(R)-A .
\end{aligned}
$$

Obviously, $B \subseteq W(1-e)$. Since $W(1-e) \subseteq J$-spec $(R)-A$, we get $A \subseteq W(e)$. In addition, $e R(1-e) \subseteq J(R)$. Therefore $J$-spec $(R)$ is strongly zero dimensional.

Conversely, assume that $J$-spec $(R)$ is strongly zero dimensional. For any $a \in R$, we see that $W(a)$ and $W(a-1)$ are disjoint closed sets of $J$-spec $(R)$. Thus, we can 
find two distinct clopen sets $U$ and $V$ such that $W(a) \subseteq U$ and $W(a-1) \subseteq V$. In light of Lemma 3.2, we have elements $e, f \in R$ such that $U=W(e)$ and $V=W(f)$, where $e R(1-e), f R(1-f) \subseteq J(R)$. If $P \in J$-spec $(R)$ and $P \notin J$-spec $(R)-W(a)$, then $P \in W(a)$, and so $P \in W(e)$. Since $W(e) \cap W(f)=W(R e R+R f R)=\emptyset$, it is easy to check that $R e R+R f R=R$. Write $\sum_{i=1}^{m} s_{i} e t_{i}+\sum_{j=1}^{n} x_{j} f y_{j}=1$. If $P \in W(f)$, then $R f R \subseteq P$, and so $\sum_{i=1}^{m} s_{i} e t_{i} \notin P$. This implies that $e \notin P$. As $e R(1-e) \subseteq J(R) \subseteq P$, we see that $1-e \in P$. This shows that $P \in W(1-e)$, a contradiction. Thus, $P \notin W(f)$. As a result,

$$
W(a-1) \subseteq W(f) \subseteq J-\operatorname{spec}(R)-W(a) .
$$

In view of Lemma 3.1, $a \in R$ is feckly clean. As a result, $R$ is feckly clean.

Recall that a ring $R$ is a Hilbert ring if every prime ideal of the ring is an intersection of maximal ideals (cf. [6]). For instance, any artinian ring, ring of integers, any polynomial ring in finitely many variables over a field. A general form states that if $R$ is a Hilbert ring, then so is any finitely generated $R$-algebra $S$.

Corollary 3.4. Let $R$ be a Hilbert ring. Then $R$ is feckly clean if and only if $\operatorname{Max}(R)$ is strongly zero-dimensional.

Proof. By hypothesis, every prime ideal contains the Jacobson radical as in the proof of Lemma 2.1. Therefore the result follows from Theorem 3.3.

Theorem 3.5. A ring $R$ is feckly clean if and only if

(1) $\operatorname{Max}(R)$ is strongly zero dimensional.

(2) Every prime ideal of $R$ containing $J(R)$ is contained in a unique maximal ideal.

Proof. Suppose that $R$ is feckly clean. In view of Theorem 2.3, $\operatorname{Max}(R)$ is strongly zero dimensional. If there exists an ideal $P \in J-\operatorname{spec}(R)$ such that $P \subseteq M \cap N$ where $M$ and $N$ are distinct maximal ideals, by virtue of Theorem 2.6, there exists an element $e \in R$ such that $e \in M, 1-e \in N$ and $e R(1-e) \subseteq J(R)$. As $e R(1-e) \subseteq$ $J(R) \subseteq P, e \in P$ or $1-e \in P$. If $e \in P$, then $e \in N$, a contradiction. If $1-e \in P$, then $1-e \in M$, a contradiction. Therefore every prime ideal of $R$ containing $J(R)$ is contained in exactly one maximal ideal.

Conversely, assume (1) and (2) hold. Then there exists a map $\varphi: J$-spec $(R) \rightarrow$ $\operatorname{Max}(R), \varphi(P)=M$, where $M$ is the unique maximal ideal such that $P \subseteq M$. Let $I$ be an ideal of $R$, we let $V_{S}(I)=\{P \in J-\operatorname{spec}(R) \mid I \subseteq P\}$ and $V_{M}(I):=\{P \in$ $\operatorname{Max}(R) \mid I \subseteq P\}$. Then $\varphi\left(V_{S}(I)\right)=V_{M}(I)$. For any disjoint closed sets $A, B \subseteq$ 
$J$-spec $(R)$, there exist two ideals $I$ and $J$ of $R$ such that $A=V_{S}(I)$ and $B=V_{S}(J)$. Thus, $\varphi(A)$ and $\varphi(B)$ are both closed. As $V_{S}(I) \cap V_{S}(J)=\emptyset, V_{S}(I+J)=\emptyset$; hence, $I+J=R$. This implies that $V_{M}(I) \bigcap V_{M}(J)=V_{M}(I+J)=V_{M}(R)=\emptyset$. That is, $\varphi(A)$ and $\varphi(B)$ are disjoint closed sets of $\operatorname{Max}(R)$. By hypothesis, $\operatorname{Max}(R)$ is strongly zero-dimensional, and so we can find disjoint clopen sets $U, V \subseteq \operatorname{Max}(R)$ such that $V_{M}(I) \subseteq U, V_{M}(J) \subseteq V$. Clearly, $A \subseteq \varphi^{\leftarrow} \varphi(A) \subseteq \varphi^{\leftarrow}(U)$ and $B \subseteq$ $\varphi^{\leftarrow} \varphi(B) \subseteq \varphi^{\leftarrow}(V)$. As in the proof of [7, Theorem 1.2], $\varphi$ is continuous; hence, $\varphi^{\leftarrow}(U)$ and $\varphi^{\leftarrow}(V)$ are clopen. For any $P \in \varphi^{\leftarrow}(U) \cap \varphi^{\leftarrow}(V)$, there exists a unique $M \in \operatorname{Max}(R)$ such that $P \subseteq M$. Hence, $M \in U \cap V$, a contradiction. This implies that $\varphi^{\leftarrow}(U) \cap \varphi^{\leftarrow}(V)=\emptyset$. Consequently, $J-\operatorname{spec}(R)$ is strongly zero-dimensional. In light of Theorem 3.3, we complete the proof.

Corollary 3.6. A ring $R$ is feckly clean if and only if

(1) $\operatorname{Max}(R)$ is strongly zero dimensional.

(2) For any distinct maximal ideals $M$ and $N$, there exist elements $a, b \in R$ such that $a \notin M, b \notin N$ and $a R b \subseteq J(R)$.

Proof. Suppose that $R$ is feckly clean. Then $\operatorname{Max}(R)$ is strongly zero dimensional by Theorem 3.5. For any distinct maximal ideals $M$ and $N$, it follows from Theorem 2.6 that there exists an element $e \in R$ such that $e \in M, 1-e \in N$ and $e R(1-e) \subseteq$ $J(R)$. Set $a=1-e$ and $b=e$. Then $a \notin M, b \notin N$ and $a R b=(1-e) R e \subseteq J(R)$.

$(2) \Rightarrow(1)$ Suppose that $P$ containing $J(R)$ is a prime ideal such that $P \subseteq M \cap N$ where $M$ and $N$ are distinct maximal ideals of $R$. Then $a R b \subseteq J(R) \subseteq P$; hence, either $a \in P$ or $b \in P$. This implies that $a \in M$ or $b \in N$, a contradiction. Therefore every prime ideal of $R$ containing $J(R)$ is contained in a unique maximal ideal. According to Theorem 3.5, we complete the proof.

Corollary 3.7. If $a+b=1$ in $R$ implies that $(1+a r) R(1+b s) \subseteq J(R)$ for some $r, s \in R$, then the following are equivalent:

(1) $R$ is feckly clean.

(2) $\operatorname{Max}(R)$ is strongly zero dimensional.

Proof. (1) $\Rightarrow(2)$ In view of Theorem 3.5, $\operatorname{Max}(R)$ is strongly zero dimensional.

$(2) \Rightarrow(1)$ Let $P \in J-\operatorname{spec}(R)$. Then $P$ is contained in some maximal ideal of $R$. Suppose that $P$ is contained in two distinct maximal ideals $M$ and $N$. Then $M+N=R$. Write $a+b=1$ with $a \in M, b \in N$. By hypothesis, there are some $r, s \in R$ such that $(1+a r) R(1+b s) \subseteq J(R)$. As $J(R) \subseteq P$, we see that either $1+a r \in P$ or $1+b s \in P$. If $1+a r \in P$, then $1=(1+a r)-a r \in M$. If $1+b s \in P$, then $1=(1+b s)-b s \in N$. In any case, we get a contradiction. Therefore every prime 
ideal of $R$ containing $J(R)$ is contained in exactly one maximal ideal. According to Theorem $3.5, R$ is feckly clean.

\section{More ExAmples}

Recall that a ring $R$ is $\pi$-regular provided that for any $a \in R$ there exists $n \in \mathbb{N}$ such that $a^{n} \in a^{n} R a^{n}$.

Theorem 4.1. If $R / J(R)$ is an abelian $\pi$-regular ring, then $R$ is feckly clean.

Proof. Let $a \in R$. In view of [13, Theorem 30.2], $R / J(R)$ is clean. Thus, there exists an idempotent $\bar{e} \in R / J(R)$ and a unit $\bar{u} \in R / J(R)$ such that $\bar{a}=\bar{e}+\bar{u}$. As units lift modulo $J(R)$, we may assume that $u \in U(R)$. Thus, we can find $r \in J(R)$ such that $a=e+(u+r)$. Clearly, $u+r \in U(R)$, and so $u+r \in R$ is a full element. As $R / J(R)$ is abelian, we see that $\bar{e}(R / J(R))(\overline{1}-\bar{e})=\overline{0}$. Therefore $e R(1-e) \subseteq J(R)$. Therefore $R$ is feckly clean.

Szász in [12] studied the ring $R$ with the property that for any $x \in R$, there exists some $n(x) \geq 2$ such that $x R x=x^{n(x)} R x^{n(x)}$. He called these rings $g s r$-rings. For instance, every strongly regular ring is a $g s r$-ring.

Corollary 4.2. Every gsr-ring is feckly clean.

Proof. Let $R$ be a $g s r$-ring. Then for any $x \in R, x^{2} \in x^{2} R x^{2}$; hence, $R$ is a $\pi$-regular ring. This implies that $R / J(R)$ is $\pi$-regular. Given $\bar{x}^{2}=\overline{0}$ in $R / J(R)$, then $x^{2} \in J(R)$. By hypothesis, $x R x=x^{2} R x^{2} \subseteq J(R)$, i.e., $(R x R)^{2} \subseteq J(R)$. As $J(R)$ is semiprime, it follows that $R x R \subseteq J(R)$, and so $x \in J(R)$. That is, $\bar{x}=\overline{0}$. This implies that $R / J(R)$ is reduced. For any idempotent $e \in R / J(R)$ and any $a \in R / J(R)$, it follows from $(e a(\overline{1}-e))^{2}=0$ that $e a(\overline{1}-e)=0$, thus $e a=e a e$. Likewise, $a e=e a e$. This implies that $e a=a e$. As a result, every idempotent in $R / J(R)$ is central, i.e., $R / J(R)$ is abelian. Therefore we complete the proof by Theorem 4.1.

Corollary 4.3. If for any $a \in R$, there exists some $n \geq 2$ such that $a^{n}-a \in J(R)$, then $R$ is feckly clean.

Proof. Let $a \in R$. Then there exists some $n \geq 2$ such that $a^{n}-a \in J(R)$, and so $\bar{a}=\overline{a^{n}}$. Therefore $R / J(R)$ is regular. By the proof of Corollary $4.2, R / J(R)$ is abelian. Hence $R$ is feckly clean by Theorem 4.1

Corollary 4.4. If $R / J(R)$ is a finite commutative ring, then $R$ is feckly clean. 
Proof. As $R / J(R)$ is a finite commutative ring, for any $a \in R$, there exist distinct $m, n \in \mathbb{N}$ such that $\overline{a^{m}}=\overline{a^{n}}$. Therefore $R / J(R)$ is $\pi$-regular, and then the result follows by Theorem 4.1 .

A ring $R$ a right (left) quasi-duo ring if every maximal right (left) ideal of $R$ is an ideal. For instance, local rings, duo rings and weakly right (left) duo rings are all right (left) quasi-duo rings (cf. [15]). Further, every abelian exchange ring is a right (left) quasi-duo ring, where a ring $R$ is an exchange ring provided that for any $a \in R$ there exists an idempotent $e \in R$ such that $e \in a R$ and $1-e \in(1-a) R$ (cf. [4] and [15]).

Theorem 4.5. Every right (left) quasi-duo exchange ring is feckly clean.

Proof. Let $R$ be a right quasi-duo exchange ring. In view of [15, Lemma 2.3], $R / J(R)$ is abelian. As $R$ is an exchange ring, then so is $R / J(R)$. In light of [4, Theorem 17.2.2], $R / J(R)$ is clean. Let $x \in R$. Then there exists an idempotent $\bar{e} \in R / J(R)$ and a unit $\bar{u} \in R / J(R)$ such that $\bar{a}=\bar{e}+\bar{u}$. We may assume that $u \in U(R)$. Thus, we can find a $r \in J(R)$ such that $a=e+(u+r)$, where $u+r \in U(R)$ is a full element. As $R / J(R)$ is abelian, we see that $e R(1-e) \subseteq J(R)$, and so $R$ is feckly clean.

Remark 4.6. Let $R=\left(\begin{array}{cc}\mathbb{Z}_{4} & \mathbb{Z}_{4} \\ 0 & \mathbb{Z}_{4}\end{array}\right)$. Then $R$ is a quasi-duo exchange ring (cf. [15]). According to Theorem 4.5, $R$ is feckly clean. Note that $R$ is not abelian.

Remark 4.7. Let $p$ and $q$ be two distinct primes other than 2. Then the ring $\mathbb{Z}_{(p)} \cap \mathbb{Z}_{(q)}$ is feckly clean, but it is not clean. As in the proof of $[1$, Proposition 16], we see that $\mathbb{Z}_{(p)} \cap \mathbb{Z}_{(q)}$ is commutative with exactly two maximal ideals. Additionally, $J(R)=p R \bigcap q R$. Therefore $R$ is a semilocal ring. That is, $R / J(R)$ is finite direct sums of division rings. Hence, $R / J(R)$ is clean. Consequently, $R$ is feckly clean. Since $(p, q)=1, \frac{p(q+1)}{p+q} \in R$. Observing that $R$ is an integral domain, the set of all idempotents in $R$ is $\left\{\frac{0}{1}, \frac{1}{1}\right\}$. As $q \nmid q+1$, we see that $\frac{p(q+1)}{p+q} \notin U(R)$. As $p \nmid(p-1) q$, we see that $\frac{p(q+1)}{p+q}-\frac{1}{1} \notin U(R)$. This shows that $\frac{p(q+1)}{p+q} \in R$ is not clean.

\section{The Commutative Case}

If $R$ is commutative then $R$ is feckly clean if and only if $R / J(R)$ is clean. In other words, in the commutative case, the difference between "feckly clean" and "clean" is that both are clean modulo the radical but in the former idempotents do 
not necessarily lift modulo the radical. The aim of this section is to investigate the necessary and sufficient conditions under which a commutative ring is feckly clean.

Theorem 5.1. Let $R$ be a commutative ring. Then the following are equivalent:

(1) $R$ is feckly clean

(2) For any $a \in R$, there exists $e \in R$ such that $a-e \in U(R), e^{2}-e \in J(R)$.

(3) For any $a \in R$, there exists $e \in R$ such that $a-e \in\left(a-a^{2}\right) R, e-e^{2} \in J(R)$.

(4) For any $a \in R$, there exists $e \in R$ such that $e \in a R, 1-e \in(1-a) R, e-e^{2} \in$ $J(R)$.

Proof. (1) $\Rightarrow(2)$ As $R$ is commutative, every full element in $R$ is invertible. Therefore for any $a \in R$, there exists an element $e \in R$ such that $a-e \in U(R), e^{2}-$ $e \in J(R)$.

$(2) \Rightarrow(3)$ For any $a \in R$, there exist $f \in R$ and $u \in U(R)$ such that $a=f+u$ and $f-f^{2} \in J(R)$. Let $e=(1-f)+\left(f-f^{2}\right) u^{-1}$. Then $e-e^{2} \equiv f-f^{2} \equiv 0(\bmod J(R))$. Hence, $e-e^{2} \in J(R)$. Further, $a-e=f+u-1+f-\left(f-f^{2}\right) u^{-1}=(u-2 f u-$ $\left.u^{2}+f-f^{2}\right)\left(-u^{-1}\right)=\left(a-a^{2}\right)\left(-u^{-1}\right) \in\left(a-a^{2}\right) R$.

$(3) \Rightarrow(4)$ For any $a \in R$, there exists $e \in R$ such that $a-e \in\left(a-a^{2}\right) R, e-e^{2} \in$ $J(R)$. Write $a-e=\left(a-a^{2}\right) s$ for some $s \in R$. Then $e=a(1-(1-a) a s) \in a R$. Further, $1-e=(1-a)(1+a s) \in(1-a) R$, as required.

$(4) \Rightarrow(1)$ For any $a \in R$, there exists $e \in R$ such that $e \in a R, 1-e \in(1-a) R, e-$ $e^{2} \in J(R)$. Write $e=a s$ and $1-e=(1-a) x$ for some $s, x \in R$. Set $f=1-e$. Then $f-f^{2}=e-e^{2} \in J(R)$. We may assume that $s e=s$ and $x(1-e)=x$. It is easy to verify that $(a-f)(s-x)=a s-a x-f s+f x=e-a x-(1-e) e s+f x=$ $e+(f-a) x=e+(f-a) f x=e+f(1-a) x=e+f=1$. Therefore $a=f+(a-f)$ with $a-f \in U(R)$, as asserted.

Corollary 5.2. Let $R$ be a commutative ring. Then the following are equivalent:

(1) $R$ is feckly clean .

(2) For any $a \in R$, there exist $n \in \mathbb{N}, e \in R$ and $u \in U(R)$ such that $a^{n}=e+u$ and $e-e^{2} \in J(R)$.

Proof. $\quad(1) \Rightarrow(2)$ It is trivial by choosing $n=1$.

$(2) \Rightarrow(1)$ For any $a \in R$, there exist $n \in \mathbb{N}, f \in R$ and $u \in U(R)$ such that $a^{n}=f+u$ and $f-f^{2} \in J(R)$. Let $e=(1-f)+\left(f-f^{2}\right) u^{-1}$. Then $e-e^{2} \in J(R)$. One easily checks that $a^{n}-e=f+u-1+f-\left(f-f^{2}\right) u^{-1}=(u-2 f u-$ $\left.u^{2}+f-f^{2}\right)\left(-u^{-1}\right)=\left(a^{n}-a^{2 n}\right)\left(-u^{-1}\right)$. This implies that $e \in a R$. Further, $1-e=\left(1-a^{n}\right)+\left(a^{n}-a^{2 n}\right)\left(-u^{-1}\right) \in(1-a) R$. Therefore $R$ is feckly clean by Theorem 5.1. 
Corollary 5.3. Let $R$ be a commutative ring. Then the following are equivalent:

(1) $R$ is feckly clean .

(2) For any $a \in R$, there exist $e \in R$ and $u \in U(R)$ such that $e=$ aue, $1-e=$ $(1-a)(-u)(1-e)$ and $e-e^{2} \in J(R)$.

Proof. (1) $\Rightarrow(2)$ For any $a \in R$, we have $a=f+v, f-f^{2} \in J(R), v \in U(R)$. Thus, $a(1-f)=v(1-f)$; hence, $1-f=v^{-1} a(1-f)$. As $1-a=1-f-v$, we have $f=\left(-v^{-1}\right)(1-a) f$. Set $e=1-f$ and $u=v^{-1}$. Then $e-e^{2} \in J(R)$. Moreover, $e=$ aue and $1-e=(1-a)(-u)(1-e)$, as required.

$(2) \Rightarrow(1)$ For any $a \in R$, there exist $e \in R$ and $u \in U(R)$ such that $e=$ aue, $1-e=(1-a)(-u)(1-e)$ and $e-e^{2} \in J(R)$. Then $e \in a R, 1-e \in(1-a) R$. According to Theorem 5.1, $R$ is feckly clean .

A Hausdorff space is a topological space in which distinct points $x$ and $y$ have disjoint open sets $U$ and $V$.

Theorem 5.4. Let $R$ be a commutative ring. Then the following are equivalent:

(1) $R$ is feckly clean .

(2) $\operatorname{Max}(R)$ is a strongly zero dimensional Hausdorff space.

(3) $\operatorname{Max}(R)$ is strongly zero dimensional and $a+b=1$ in $R$ implies that $(1+a r)(1+b s) \in J(R)$ for some $r, s \in R$.

(4) $a+b=1$ in $R$ implies that $1+$ ar $\in e R, 1+b s \in(1-e) R$ where $e-e^{2} \in J(R)$.

Proof. (1) $\Leftrightarrow(2)$ In view of [7, Theorem 1.3], $\operatorname{Max}(R)$ is an Hausdorff space if and only if every prime ideal of $R$ containing $J(R)$ is contained in a unique maximal ideal, and proving (2) by Theorem 3.5.

$(1) \Rightarrow(3)$ In view of Theorem 3.5, $\operatorname{Max}(R)$ is strongly zero dimensional. Given $a+b=1$ in $R$, there exists $u \in U(R)$ such that $a=e+u$ where $e-e^{2} \in J(R)$. It is easy to verify that

$$
\begin{aligned}
& \left(1+\frac{a}{e-a}\right)\left(1-\frac{b}{e-a}\right) \\
= & 1-\frac{b}{e-a}+\frac{a}{e-a}-\frac{a b}{(e-a)^{2}} \\
= & \frac{e^{2}-e}{(e-a)^{2}} \\
\in & J(R) .
\end{aligned}
$$

Choose $r=\frac{1}{e-a}$ and $t=-\frac{1}{e-a}$. Then $(1+a r)(1+b s) \in J(R)$.

$(3) \Rightarrow(1)$ Let $P$ be a prime ideal of $R$ such that $J(R) \subseteq P$ and $P$ is contained in two distinct maximal ideals $M$ and $N$. Write $a+b=1$ with $a \in M, b \in N$. By hypothesis, there are some $r, s \in R$ such that $(1+a r)(1+b s) \in J(R)$. As $J(R) \subseteq P$, we see that either $1+a r \in P$ or $1+b s \in P$. If $1+a r \in P$, then $1=(1+a r)-a r \in M$. If $1+b s \in P$, then $1=(1+b s)-b s \in N$. In any case, we 
get a contradiction. Therefore every prime ideal of $R$ containing $J(R)$ is contained in exactly one maximal ideal. According to Theorem $3.5, R$ is feckly clean.

(1) $\Rightarrow$ (4) Suppose that $R$ is feckly clean and $a+b=1$ in $R$. In view of Theorem 5.1, there exists an element $f \in R$ such that $f \in a R, 1-f \in(1-a) R$ and $f^{2}-f \in J(R)$. Write $1-f=-(1-a) s$ and $f=-a r$ for some $r, s \in R$. Then $1+b s=f$ and $1+a r=1-f$. Set $e=1-f$. Then $e^{2}-e=f^{2}-f \in J(R)$. Further, $1+a r \in e R$ and $1+b s \in(1-e) R$, as required.

$(4) \Rightarrow(1)$ is trivial by Corollary 2.7 .

Corollary 5.5. A commutative ring $R$ is feckly clean if and only if $\operatorname{Max}(R)$ is strongly zero-dimensional and $J$-spec $(R)$ is normal.

Proof. In view of [7, Theorem 1.3], $J$-spec $(R)$ is normal if and only if $\operatorname{Max}(R)$ is a Hausdorff space, and so the result follows by Theorem 5.4.

Recall that a commutative ring $R$ is a $p m$ (Gelfand) ring provided that each prime ideal of $R$ is contained in exactly one maximal ideal. In [1, Corollary 4], Anderson and Camillo proved that every commutative clean ring is always a $\mathrm{pm}$ ring.

Theorem 5.6. Let $R$ be a commutative ring. Then the following are equivalent:

(1) $R$ is clean.

(2) $R$ is feckly clean and $R$ is a pm ring.

(3) $R$ is feckly clean ring in which $a+b=1$ implies that $(1+a r)(1+b s)=0$ for some $r, s \in R$.

Proof. (1) $\Rightarrow(2)$ Clearly, $R$ is feckly clean. According to [1, Corollary 4$], R$ is a pm ring.

$(2) \Rightarrow(1)$ For any disjoint closed sets $A$ and $B$ of $\operatorname{Max}(R)$, it follows from Theorem 2.3 that there exists an element $e \in R$ such that $A \subseteq V(e), B \subseteq V(1-e)$ and $e R(1-e) \subseteq J(R)$. As $e R(1-e) \subseteq J(R)$, we get $\operatorname{Max}(R)=V(e) \bigsqcup V(1-e)$; hence that $V(1-e)$ and $V(e)$ are clopen sets of $\operatorname{Max}(R)$. Therefore $\operatorname{Max}(R)$ is strongly zero-dimensional. According to [4, Corollary 17.1.14], $R$ is strongly clean.

$(2) \Leftrightarrow(3)$ According to [5, Theorem 4.4], $R$ is a pm ring if and only if $a+b=1$ implies that $(1+a r)(1+b s)=0$ for some $r, s \in R$, and so the result follows.

Corollary 5.7. Let $X$ be a topological space. Then the following are equivalent:

(1) $C(X)$ is clean.

(2) $C(X)$ is feckly clean. 
Proof. In view of [3], $C(X)$ is a pm ring. Thus, the proof is true by Theorem 5.6.

Corollary 5.8. Let $R$ be a commutative ring. Then the following are equivalent:

(1) $R$ is $\pi$-regular.

(2) $R$ is feckly clean ring in which every prime ideal is maximal.

Proof. $(1) \Rightarrow(2)$ In view of Theorem $4.1, R$ is feckly clean. According to $[6$, Corollary 2.8], every prime ideal of $R$ is maximal.

$(2) \Rightarrow(1)$ In light of Theorem $3.5, \operatorname{Max}(R)$ is strongly zero-dimensional. Therefore we complete the proof by $[6$, Theorem 2.3].

\section{ACKNOWLEDGEMENTS}

The authors are grateful to the referee for his/her helpful suggestions which correct many errors in the first version and lead the new one more clear. This research was supported by the Natural Science Foundation of Zhejiang Province (LY13A010019) and the Scientific and Technological Research Council of Turkey (2221 Visiting Scientists Fellowship Programme).

\section{REFERENCES}

[1] D.D. Anderson and V.P. Camillo, Commutative rings whose elements are a sum of a unit and idempotent, Comm. Algebra, 30(2002), 3327-3336.

[2] W.D. Burgess and R. Raphael, On commutative clean ring and pm rings, Contemporary Math., Topology and Its Applications, 137(2004), 21-37.

[3] B. Banaschewski, Rings theory and pointfree topology, Topology and Its Applications, 137(2004), 21-37.

[4] H. Chen, Rings Related Stable Range Conditions, Series in Algebra 11. Hackensack, NJ: World Scientific. 2011.

[5] M. Contessa, On pm-ring, Comm. Algebra, 10(1982), 93-108.

[6] A. Kaucikas and R. Wisbauer, Noncommutative Hilbert rings, J. Algebra Appl., 03, 437 (2004). DOI: 10.1142/S0219498804000964.

[7] G.D. Marco and A. Orsatti, Commutative rings in which every prime ideal is contained in a unique maximal ideal, Proc. Amer. Math. Soc., 30(1971), 459-466.

[8] W.W. McGovern, Neat rings, J. Pure and Applied Algebra, 205(2006), 243-265.

[9] W.W. McGovern, A characterization of commutative clean rings, Int. J. Math. Game Theory Algebra, 15(2006), 403-413.

[10] W.K. Nicholson, Clean rings: a survey, Advances in Ring Theory, World Sci. Publ., Hackensack, NJ, 2005, 181-198.

[11] K. Samei. Clean elements in commutative reduced rings, Comm. Algebra, 32(2004), $3479 \mathrm{C} 3486$. 
[12] F.A. Szász, On generalized subcommutative regular rings, Monatshefte für Mathematik, $\mathbf{7 7}(1973), 67-71$.

[13] A.A. Tuganbaev, Rings Close to Regular, Kluwer Academic Publishers, Dordrecht, Boston, London, 2002.

[14] K. Varadarajan, Clean, almost clean, potent commutative ring, J. Algebra Appl., 6(2007), 671-685.

[15] H.P. Yu, On quasi-duo rings, Glasg. Math. J., 37 (1995), 21-31. 